\title{
In silico prediction reveals the existence of potential bioactive neuropeptides produced by the human gut microbiota
}

\author{
Aitor Blanco-Míguez ${ }^{\mathrm{a}, \mathrm{b}, \mathrm{c}}$, Florentino Fdez-Riverola ${ }^{\mathrm{a}, \mathrm{b}, \mathrm{d}}$, Anália Lourenço ${ }^{\mathrm{a}, \mathrm{b}, \mathrm{d}, \mathrm{e}}$, Borja Sánchez ${ }^{\mathrm{c}, *}$ \\ ${ }^{\text {a }}$ ESEI: Escuela Superior de Ingeniería Informática, University of Vigo, Edificio Politécnico, Campus Universitario As Lagoas $s / n, 32004$ Ourense, Spain \\ ${ }^{\mathrm{b}}$ CINBIO - Centro de Investigaciones Biomédicas, University of Vigo, Campus Universitario Lagoas-Marcosende, 36310 Vigo, Spain \\ ${ }^{\mathrm{c}}$ Department of Microbiology and Biochemistry of Dairy Products, Instituto de Productos Lácteos de Asturias (IPLA), Consejo Superior de Investigaciones Científicas \\ (CSIC), Paseo Río Linares S/N, 33300 Villaviciosa, Asturias, Spain \\ ${ }^{\mathrm{d}}$ SING Research Group, Galicia Sur Health Research Institute (IIS Galicia Sur), SERGAS-UVIGO, Hospital Álvaro Cunqueiro, 36312 Vigo, Spain

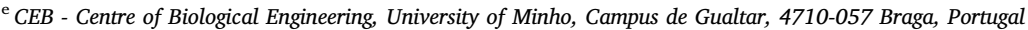

\section{A R T I C L E I N F O}

\section{Keywords:}

Gut microbiota

Bioactive peptides

Metaproteomes

Peptidomes

Neuropeptides

Anti-inflammatory

Immunomodulatory

Microbiome

\begin{abstract}
A B S T R A C T
This work reports on a large-scale potential neuropeptide activity screening in human gut microbiomes deposited in public databases. In our experimental approach, the sequences of the bioactive peptides collected in the MAHMI database, mainly predicted as immunomodulatory or antitumoral, were crossed with those of the neuroactive/digestive peptides. From 91,325,790 potential bioactive peptides, only 581 returned a match when crossed against the 5949 neuroactive peptides from the NeuroPep database and the 15 digestive hormones. Relevant bacterial taxa, such as Ruminococcus sp., Clostridium sp. were found among the main producers of the matching sequences, and many of the matches corresponded to adiponectin and the hormone produced by adipocites, which is involved in glucose homeostasis. These results show, for the first time, the presence of potentially bioactive peptides produced by gut microbiota members over the nervous cells, most notably, peptides with already predicted immunomodulatory or anti-inflammatory activity. Classical (Lactobacillus sp.) and next-generation (Faecalibacterium sp.) probiotics are shown to produce these peptides, which are proposed as a potential mechanism of action of psychobiotics. Our previous experimental results showed that many of these peptides were active when incubated with immune cells, such as dendritic cells, so their effect over the nervous system innervating the gut mucosa holds significant potential and should be explored.
\end{abstract}

\section{Introduction}

The gut microbiota and the brain interact with each other through several bidirectional signalling pathways clustered in the so-called gutbrain axis, in which a number of cell types and neuroactive metabolites act as messengers and play important mediator roles (Holzer, 2016). Among them, neuropeptides are small proteinaceous substances produced by nervous cells, which are released in a regulated fashion and act on neural lineages, such as neurons and glial cells, as well as nonneuronal target cells, such as glandular or muscular cells (Burbach, 2010). It is well-known that a vast number of neuropeptides are produced by central and peripheral neurons alongside with endocrine cells in the gastrointestinal tract and other endocrinologically active organs (Burbach, 2010; Kastin, 2013; Strand, 1999). Neuropeptides such as substance P, calcitonin gene-related peptide, neuropeptide Y (NPY), vasoactive intestinal polypeptide, somatostatin and corticotropin-releasing factor (CRF) are also likely to play a role in the bidirectional gut-brain communication, influencing the activity of the gastrointestinal microbiota and their interaction through the gut-brain axis (Holzer \& Farzi, 2014). Other biologically active peptides, which are released to the blood stream and depend on the presence/absence of nutrients along the digestive tract, also function as gut hormones. Gut hormones and neuropeptides form families of closely related peptides. For example, the peptide YY (PYY) and the pancreatic polypeptide are closely related with the neuropeptide Y (NPY) (Holzer, 2016). Given that neuropeptides and gut hormones may target the same cell membrane receptors, the two messenger roles often converge in the same or similar biological implications.

The first neuropeptide, i.e. substance P, was discovered by van Euler and Gaddum in 1931 (Euler \& Gaddum, 1931) and sequenced in 1971 (Chang, Leeman, \& Niall, 1971). Since then, there has been a tremendous increase in the number of identified neuropeptides. However, there are only three neuropeptide databases publicly available, i.e. Neuropeptides (Burbach, 2010), NeuroPedia (Kim, Bark, Hook, \&

\footnotetext{
* Corresponding author.

E-mail address: borja.sanchez@csic.es (B. Sánchez).
} 
Bandeira, 2011) and Neuropep (Wang et al., 2015) (Burbach, 2010; Kim et al., 2011; Wang et al., 2015), of which the NeuroPep database presents the most complete collection. The current version of NeuroPep (Feb 10th 2015) holds 5949 non-redundant neuropeptides, originating from 493 organisms and belonging to 65 different neuropeptide families (http://isyslab.info/NeuroPep/).

The gut microbiota is important for educating the immune system to recognise foreign antigens and to tolerate commensal microbes and other diet-derived molecules (Lathrop et al., 2011). The gut microbes can modulate, tune and tame the host immune response (Sathyabama, Khan, \& Agrewala, 2014). Public databases, such as MAHMI, aim to facilitate the work of those scientific teams researching molecular interactions between the human host and the intestinal microbiota (Blanco-Míguez, Gutiérrez-Jácome, Fdez-Riverola, Lourenço, \& Sánchez, 2017). Currently, the MAHMI database compiles 91,325,790 peptides, extracted from extracellular proteins of the human microbiome and selected according to their immunomodulatory or antiproliferative bioactivity potential.

In the context of the microbiota-gut-brain axis, neuropeptides such as NPY have a distinctive impact on the immune function, within and outside the gastrointestinal tract (Bedoui, von Hörsten, \& Gebhardt, 2007; Dimitrijević \& Stanojević, 2013; Peter Holzer, Reichmann, \& Farzi, 2012; Wheway, Herzog, \& Mackay, 2007). Till date, to the best of our knowledge, the potential existence of neuropeptides encrypted in the proteins produced by human intestinal microbes has not been explored. This lack of information contrasts with existing knowledge on other neurotransmitters produced by the human gut microbiota, such as serotonine or GABA (Cryan \& Dinan, 2012; Nicholson et al., 2012).

Within this scenario, our previous results showing the existence and in vitro activity of immunomodulatory peptides encrypted in the human gut metaproteome (Hidalgo-Cantabrana et al., 2017), motivated the hereby exploration of neuropeptides encrypted in the protein complement of our human microbiome. In particular, the aim of this prospective work was to conduct an in silico, large-scale screening of potential neuroactive peptides encrypted in the human gut metaproteome. Such analysis was based on the collection of bioactive peptides in the MAHMI database and the collection of neuroactive peptides in the NeuroPep database, complemented with a set of the major gut hormones/peptides. Main results and future research avenues are discussed in the next sections.

\section{Materials and Methods}

\subsection{Neuropeptide and digestion hormones data retrieval}

An in house script was used to retrieve the amino acid sequences of the 5949 neuropeptides in the NeuroPep database (Wang et al., 2015) (http://isyslab.info/NeuroPep/home.jsp). Associated record metadata, such as the name of the neuropeptide, the source organism, neuropeptide family, and UniProt cross-reference (UniProt Consortium, 2018), were also retrieved. Moreover, a manual search in UniProt enabled the compilation of data on 17 digestive hormones. All data is listed in Supplementary Material S1.

\subsection{Bioactive peptides data retrieval}

All sequence data from the potential bioactive section of the MAHMI database (Blanco-Míguez et al., 2017) were retrieved upon email request to the authors. A total of $91,325,790$ potential bioactive peptides were available at the time of this study.

\subsection{Sequence comparison}

The 5949 neuropeptides were divided in two sets: a set of 283 neuropeptides from Homo sapiens and a set of 5665 neuropeptides obtained from other organisms. Therefore, three amino acid BLAST databases were created, one for each of these sets and a third database for the digestive hormones. The three BLAST databases were created using the makeblastdb command of the BLAST + suite of commands (Camacho et al., 2009). The -dbtype prot and -parse_seqids arguments were specified for the correct generation of the databases.

The 91,325,790 potential bioactive peptides retrieved from the MAHMI database were matched against the three previous databases using the BLASTp alignment tool (Madden, 2013). Successful alignments, i.e. those with an expected value (e-value) less than $1 \mathrm{e}^{-5}$, are shown in Supplementary Material S2. Additionally, Supplementary Material S3 shows the same data with a similarity threshold value of $50 \%$, with the aim to reduce non-functional false positive results in the successful alignments.

\subsection{Motif discovery}

The Multiple Em for Motif Elicitation (MEME) Suite 5.0.3 (Bailey et al., 2009) was employed to discover common motifs shared by the predicted neuropeptides. Classic discovery mode was selected to find motifs between 6 and 50 amino acids length. The predicted motif is presented in Supplementary Material S4.

\subsection{Genus prediction}

In order to predict the bacterial genus, the amino acid sequences from the 581 predicted neuropeptide/encrypted peptides retrieved in our analysis were aligned against the NCBI non-redundant database (nr) (Pruitt, Tatusova, Brown, \& Maglott, 2012) using BLASTp. Genus prediction results are listed in Supplementary Material S5. Genera that contribute to the pool of potential bioactive peptides in less than $1 \%$ are shown in the Others group.

\subsection{Enriched pathways identification in genus producing potential neuropeptides}

The pathways enriched by genus producing potential neuropeptides was investigated using the PICRUSt tool version 1.1.0 (Langille et al., 2013). PICRUSt requires OTU abundances mapped to Greengenes OTU IDs as input for the prediction. From the 62 genus predicted, only 33 were mapped to the Greengenes 15.3 database (McDonald et al., 2012). Using a mock community from derived from a gut microbiota as control sample, three synthetic communities were created enriching the relative abundance of the neuroactive peptide producing genus. The abundance of the predicted genus in these synthetic communities was increased 2, 10 and 100 fold with respect to the OTU with greater relative abundance in the control community (available as linked research data). KEGG annotations were selected to report pathway predictions. Results of the three higher levels within the pathway hierarchy were reported in Supplementary Material S6. Pathways with changes greater than $0.3 \%$ were selected as significative.

\section{Results and discussion}

The human intestinal microbiota plays a central role in regulating not only proper immune system maturation, but also brain function through interaction with the nervous system (Rao \& Gershon, 2016; Rooks \& Garrett, 2016). Moreover, different scientific studies have identified microbiota-derived bioactive molecules, which exert their immunomodulatory effects in the gut mucosa as well as in relatively distant body locations, such as the brain (Blander, Longman, Iliev, Sonnenberg, \& Artis, 2017). Among other, the following molecules are worth of mention: i) the short chain fatty acids with anti-inflammatory properties, such as butyrate, are produced through fermentative metabolism from dietary fibre by many Firmicutes; ii) the polysaccharide A of Bacteroides fragilis has an impact on the expansion of Treg cell populations; iii) the dipeptide aldehydes produced by anaerobe 

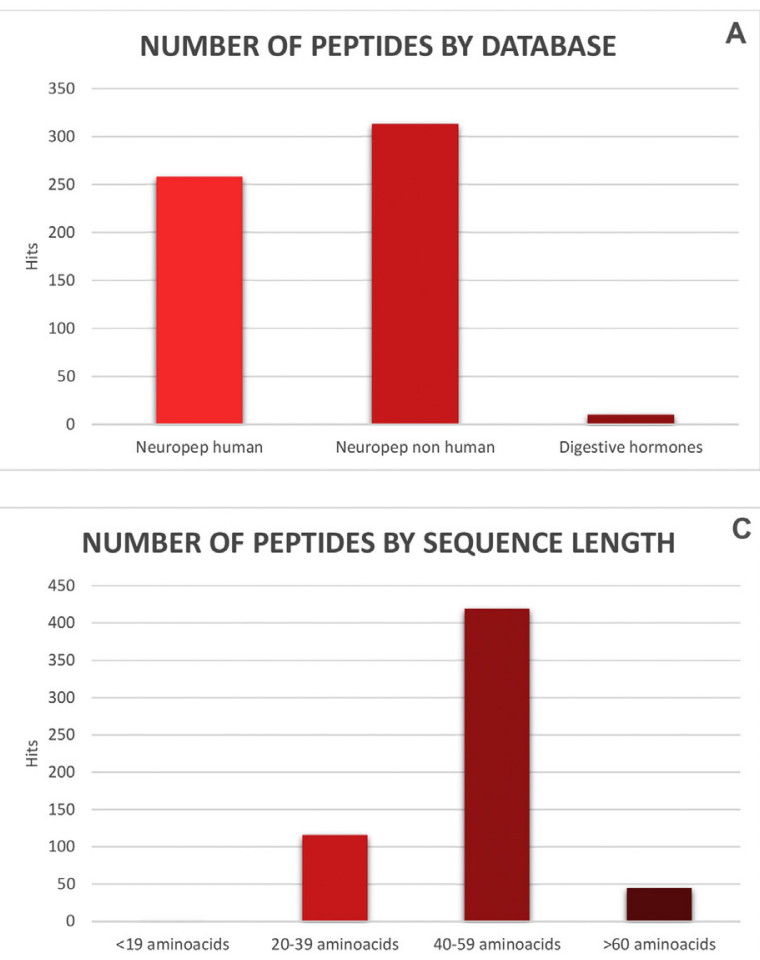

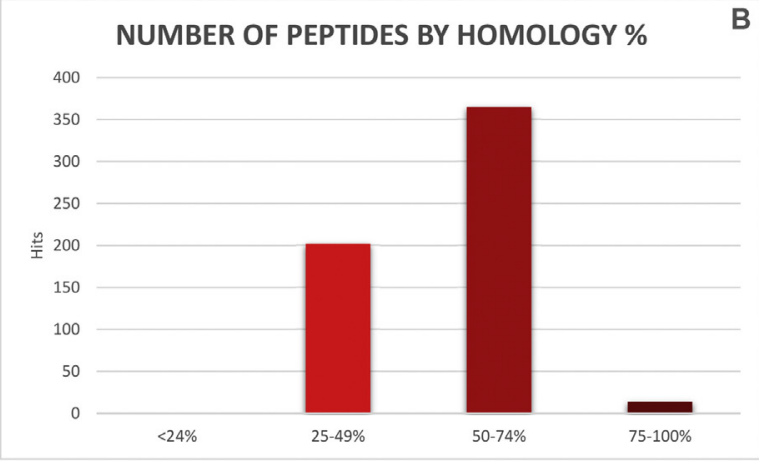

\% PEPTIDES BY MATCHED BIOACTIVITIES

D

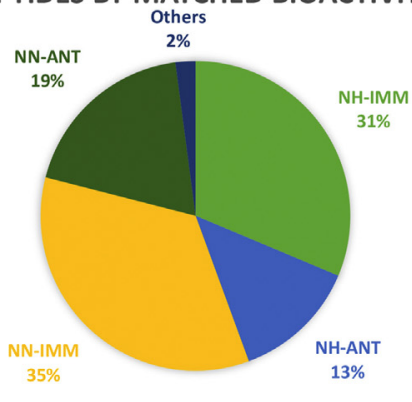

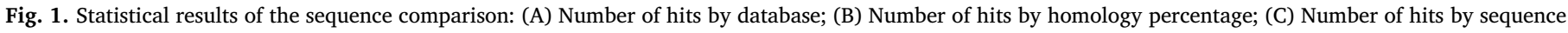

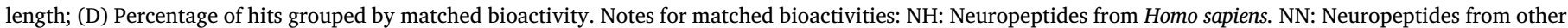
organisms. ANT: Anti-inflammatory. IMM: Immunomodulatory.

Firmicutes taxa, which interact with an uncharacterised chatepsin target that blocks the function of the innate immune system; and, iv) the S-layer protein from Lactobacillus acidophilus that interaction directly with dendritic cells promoting IL-10 production (Furusawa et al., 2013; Guo et al., 2017; Lightfoot et al., 2015; Mazmanian, Round, \& Kasper, 2008). However, there is still little understanding about how a given commensal microbiota modulates host-immune function. Many of the molecular mechanisms of action by which these bacteria exert immunomodulatory or other effects remain unknown or poorly characterised. This includes the potential presence of encrypted peptides in the human gut metaproteome with neuroactive bioactivity once released by the action of gastrointestinal proteases. To our knowledge this has not been explored so far.

Fig. 1 depicts the main results obtained after matching the potential immunomodulatory and antitumoral peptides against the three reference databases of i) human neuropeptides, ii) non-human neuropeptides and iii) digestive hormones. Only a very low percentage of the MAHMI peptides, 581 out of more than 91 million peptides, could be matched (Supplementary Material S2). In particular, 258 peptides got a match in the human neuropeptide database $(0.000283 \%), 313$ in the non-human neuropeptide database $(0.000343 \%)$ and 10 in the group of digestive hormones $(0.000011 \%$ ) (Fig. 1A). Considering homology, 202 peptides returned similarity values between 25 and 49\% (34.77\%), 365 of the values were in the range of $50-74 \%(62.82 \%)$ and only 14 peptides had similarity values between 75 and 100\% (2.41\%) (Fig. 1B). Finally, the distribution of peptides by sequence length (Fig. 1C) and by matched bioactivity (Fig. 1D) revealed some interesting patterns. For example, most of the matches of potential immunomodulatory or antiinflammatory were related to human or non-human neuropeptides (98\%), even applying a similarity threshold of 50\% (Supplementary Material S3).

Fig. 2A represents the distribution of matched peptides according to the intestinal bacteria genus that produces the source protein. An interesting result is that the most important producers of potential neuropeptides are members of the genera Ruminococcus (14\%) and Clostridium (10\%), as well as an uncharacterised Firmicutes genus (7\%). These genera represent important and very abundant taxa (many not yet cultured) of the human gut microbiota with the ability to expand Treg cell populations, favouring intestinal homeostasis (Atarashi et al., 2013). In the same way, other Firmicutes genera, such as Blautia sp. and Faecalibacterium sp., are the source of $4 \%$ of the analysed bioactive peptides. This is again important because species belonging to these genera are butyrate producers, a well-known anti-inflammatory metabolite that also favours intestinal homeostasis (Machiels et al., 2013). Moreover, strains belonging to the genus Faecalibacterium often exhibit decreased numbers in the framework of Inflammatory Bowel Disease and, most notably, their absence is considered a biomarker of inflamed mucosa (Sokol et al., 2008). Noteworthy, a healthy individual harbours grams of bacteria belonging to the above mentioned Firmicutes genera. Therefore, high local concentrations of these potential bioactive peptides can be originated after the action of gastrointestinal endoproteases over these gut bacteria. Surprisingly, Bacteroidetes, i.e. the genus from the other main phyla of the human gut microbiota, showed a limited percentage of bioactive peptides in the present analysis: Bacteroides sp. $3 \%$ and Prevotella $2 \%$. Other interesting genera producing potential neuroactive peptides are Lactobacillus (3\%), Streptococcus (3\%) and Escherichia (2\%).

An over-representation of these genera (10 or 100 -fold) within a mock microbiota produced changes in pathways related to metabolism, cellular processes, and environmental and genetic information processing levels (Supplementary Material S6). Regarding to the metabolism pathway level, the carbohydrate metabolism (1.1-1.2\%) and the glycan biosynthesis and metabolism were over-expressed $(0.8 \%)$, while nucleotide metabolism was found under-expressed $(0.4 \%)$. At the cellular processes pathway level, cell motility pathway was found under-expressed $(0.9 \%)$. At the genetic information processing level, replication and repair $(1.2 \%)$, transcription $(0.4 \%)$ and translation $(0.6 \%)$ were pathways were under-represented. Respecting the environmental 

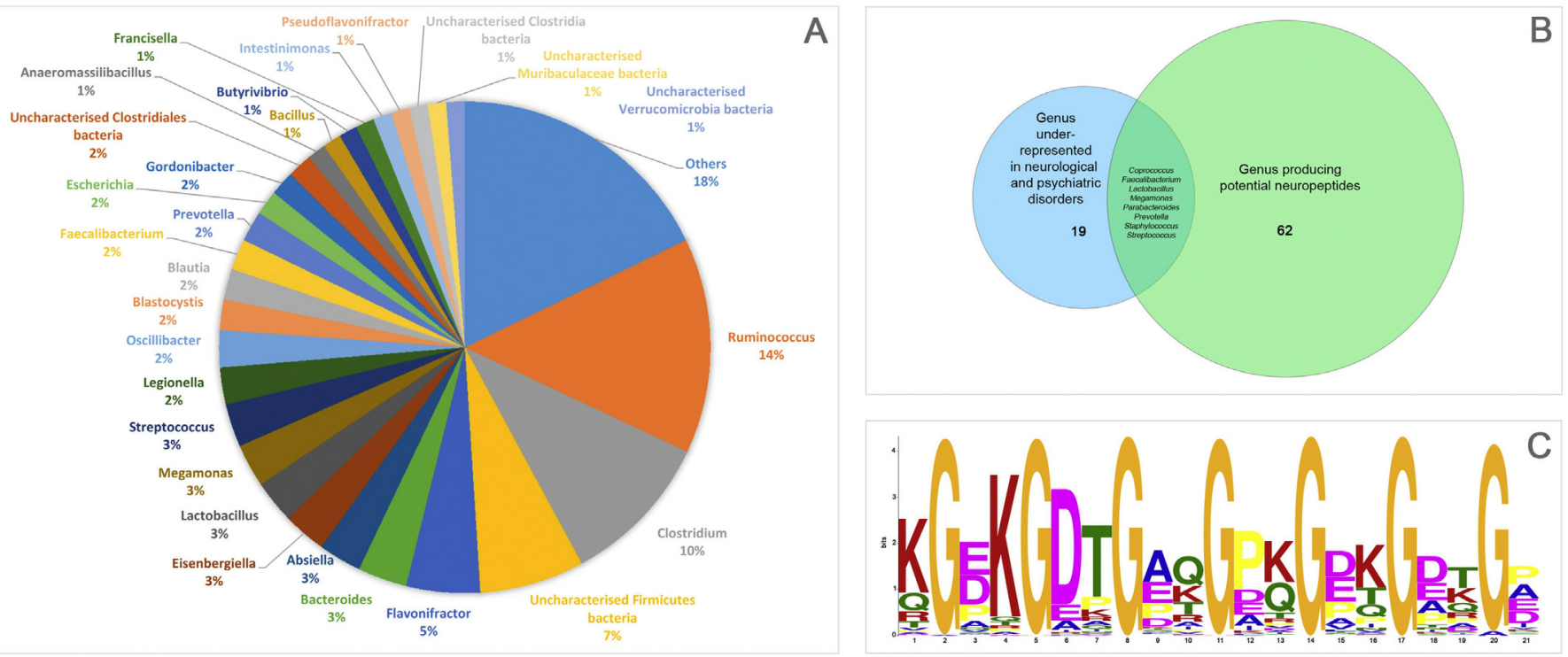

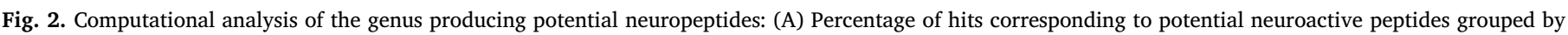

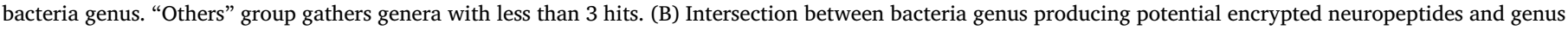
under-represented in neurological and psychiatric disorders. (C) Motif shared by 202 out of the 235 potential neuropeptides detected in our analysis.

information processing level, at the membrane transport level the phosphotransferase system pathway was found over-represented $(0.4 \%)$, but transcription machinery $(0.3-0.4 \%)$, $\mathrm{ABC}$ transporters (0.6\%-) transporters $(0.9 \%)$ pathways were under-represented. Moreover, the Sporulation pathway of the Cellular Process and Signalling level was also under-represented $(0.5 \%)$.

Some strains belonging to these genera, notably to the genus Lactobacillus, are considered probiotics given the beneficial effects shown over human health in clinical trials, and these include the socalled psychobiotics (Dinan, Stanton, \& Cryan, 2013). Psychobiotics are defined as live organisms that, when ingested in adequate amounts, produce a health benefit in patients suffering from psychiatric illness (Dinan et al., 2013). Psychobiotics are known to produce neurotransmitters in the gut, such as gamma-aminobutyric acid and serotonin, which are able to reach the nervous system and act on the braingut axis (Rao \& Gershon, 2016). Our results propose bacterial neuropeptides as additional molecular mediators of the mechanism of action of psychobiotics.

Fig. 2B illustrated common bacterial genus between those producing potential neuropeptides $(n=62)$ and those reported as under-represented in neurological and psychiatric disorders $(n=19)$ (Fung, Olson, \& Hsiao, 2017). Only microbiota changes at genus level are retrieved from the Fung et al. review. Coprococcus, Faecalibacterium, Lactobacillus, Megamonas, Parabacteroides, Prevotella, Staphylococcus and Streptococcus genus were found in both sets. However, a more severe filtering of the results applying a sequence similarity threshold of $50 \%$, revealed that matches belonging to Coprococcus and Parabacteroides genus could be indeed non-functional false-positives (Supplementary Material S3). These results suggest that the potential neuropeptides produced by these bacteria genus might have a role on these disorders, although this remains a merely speculative hypothesis that will require extensive experimentation.

Interestingly, most of the immunomodulatory/anti-inflammatory peptides were matched against adiponectin (235) and, in a much lower extent, nicotinamide phosphoribosiltransferase (12). Other human neuropeptides/gastric hormones with matches in the MAHMI database are the kininogen-1 (5), the peptide YY (3), the angiotensinogen (3), the leptin (2), the fibroblast growth factor 1pad (2) and the glucagon-like peptide 1 (1) (Table 1). Adiponectin is the main hormone secreted by adipocytes; reduction on the levels of this peptide has been linked with obesity-related and cardiovascular diseases (Achari \& Jain, 2017).
Other cell types, such as myocytes, also secrete adiponectin. Adiponectin circulates in different molecular mass forms, with different degree of multimerisation, and its administration has been related to antiatherogenic/anti-inflammatory effects (Fantuzzi, 2005). In some studies, administration of this peptide has also been linked to an insulinsensitising effect and a decrease in body weight (Fonseca, 2003).

As can be seen in Fig. 2C, 202 out of the 235 different predicted neuropeptides shared a common motif of 21 amino acids length. Similar results were obtained with a Clustal $\Omega$ alignment of the hits (Supplementary Material S7). Of these 202 peptides, 168 matched against the collagen-like domain of adiponectin (Supplementary Material S8). Agreeing with the obtained motif, collagen-like proteins are constituted by repetitions of the triplet Gly-X-Y, where $\mathrm{Y}$ is often a prolyl or lysyl residue (Herbert, Williams, Cooper, \& Brimble, 2012). Many collagenlike proteins have shown biomedical potential as anticancer agents, in particular as angiogenesis inhibitors. For example, the collagen-like protein endostatin was developed as anticancer drug for non-small cell lung cancer (O'Reilly et al., 1997). Adiponectin has been proposed as a drug candidate for the treatment of liver disease, type II diabetes, hypertension and other obesity-related disorders such as obesity-related breast cancer (Matsuzawa, 2010; Shetty, Kusminski, \& Scherer, 2009; Trujillo \& Scherer, 2005; Wang et al., 2005; Wang, Xu, Knight, Xu, \& Cooper, 2002). Whether potential neuroactive peptides derived from the gut microbiota might have complementary physiological roles to those ascribed to adiponectin, will centre our scientific efforts during next years.

As a whole, the proposed in silico pipeline shows, for the first time ever, the presence of potential neuroactive peptides encrypted in the proteomes of main members of the human gut microbiota, such as Ruminococcus sp. or Clostridium sp., and in Lactobacillus sp., a genus containing probiotic strains. Whether these peptides are able to modulate the function of neurons located in the gut mucosa can be only hypothesised and will require further experimental testing, as we previously did for some immunomodulatory peptides encrypted also in the human gut metaproteome (Hidalgo-Cantabrana et al., 2017).

\section{Conclusions}

This work presents a first, in silico evidence of the existence of peptides with a double bioactivity: neuropeptide or digestive-related, and immunomodulatory or anti-inflammatory. Noteworthy, the 
Table 1

Neuropeptides and digestion hormones detected in the sequence comparison.

\begin{tabular}{|c|c|c|c|c|c|}
\hline Name & Organism & Hits & Family & Uniprot id & Length \\
\hline Adiponectin & Homo sapiens & 235 & NA & ADIPO_HUMAN & 226 \\
\hline Adiponectin & Bos taurus & 115 & NA & ADIPO_BOVIN & 223 \\
\hline Adiponectin & Mus musculus & 110 & NA & ADIPO_MOUSE & 230 \\
\hline Nicotinamide phosphoribosyltransferase & Homo sapiens & 12 & NAPRTase & NAMPT_HUMAN & 491 \\
\hline Nicotinamide phosphoribosyltransferase & Sus scrofa & 10 & NAPRTase & NAMPT_PIG & 491 \\
\hline Nicotinamide phosphoribosyltransferase & Mus musculus & 10 & NAPRTase & NAMPT_MOUSE & 491 \\
\hline Nicotinamide phosphoribosyltransferase & Rattus norvegicus & 10 & NAPRTase & NAMPT_RAT & 491 \\
\hline Alpha-1-antitrypsin homolog & Cyprinus carpio & 6 & Serpin & A1AT_CYPCA & 353 \\
\hline Kininogen-1 & Homo sapiens & 5 & Cystatin & KNG1_HUMAN & 626 \\
\hline Alpha-1-antiproteinase & Callosciurus caniceps & 5 & Serpin & A1AT_CALCN & 388 \\
\hline Alpha-1-antiproteinase $\mathrm{F}$ & Cavia porcellus & 5 & Serpin & A1AF_CAVPO & 381 \\
\hline Alpha-1-antiproteinase S & Cavia porcellus & 5 & Serpin & A1AS_CAVPO & 381 \\
\hline Alpha-1-antitrypsin-like protein CM55-MS & Tamias sibiricus & 5 & Serpin & ALMS_TAMSI & 389 \\
\hline Alpha-1-antitrypsin-like protein CM55-ST & Tamias sibiricus & 5 & Serpin & ALST_TAMSI & 389 \\
\hline Inositol hexakisphosphate and diphosphoinositol-pentakisphosphate kinase 2 & Mus musculus & 4 & Glucagon & VIP2_MOUSE & 1129 \\
\hline Alpha-1-antitrypsin & Sus scrofa & 4 & Serpin & A1AT_PIG & 397 \\
\hline Alpha-1-antitrypsin-like protein CM55-MM & Tamias sibiricus & 4 & Serpin & ALMM_TAMSI & 389 \\
\hline Alpha-1-antitrypsin-like protein CM55-SI & Tamias sibiricus & 4 & Serpin & ALSI_TAMSI & 389 \\
\hline Peptide YY & Homo sapiens & 3 & NA & P10082 & 36 \\
\hline Angiotensinogen & Homo sapiens & 3 & Serpin & ANGT_HUMAN & 452 \\
\hline Leptin & Homo sapiens & 2 & NA & P41159 & 146 \\
\hline Fibroblast growth factor 1 & Homo sapiens & 2 & NA & P05230 & 140 \\
\hline Alpha-1-antitrypsin & Meriones unguiculatus & 2 & Serpin & A1AT_MERUN & 382 \\
\hline Alpha-1-antitrypsin-like protein GS55-LT & Spermophilus tridecemlineatus & 2 & Serpin & ALLT_SPETR & 392 \\
\hline Alpha-1-antitrypsin-like protein GS55-MS & Spermophilus tridecemlineatus & 2 & Serpin & ALMS_SPETR & 389 \\
\hline Glucagon-like peptide 1 & Homo sapiens & 1 & NA & $\mathrm{P} 01275$ & 37 \\
\hline Pancreatic hormone & Homo sapiens & 1 & NA & P01298 & 36 \\
\hline Big gastrin & Homo sapiens & 1 & NA & P01350 & 34 \\
\hline Resistin-like alpha & Mus musculus & 1 & Resistin/FIZZ & RETNA_MOUSE & 88 \\
\hline Plasma serine protease inhibitor & Bos taurus & 1 & Serpin & IPSP_BOVIN & 380 \\
\hline Angiotensinogen & Gorilla gorilla gorilla & 1 & Serpin & ANGT_GORGO & 452 \\
\hline Corticosteroid-binding globulin & Homo sapiens & 1 & Serpin & CBG_HUMAN & 383 \\
\hline Serpin A12 & Homo sapiens & 1 & Serpin & SPA12_HUMAN & 394 \\
\hline Serpin I2 & Homo sapiens & 1 & Serpin & SPI2_HUMAN & 387 \\
\hline Corticosteroid-binding globulin & Oryctolagus cuniculus & 1 & Serpin & CBG_RABIT & 383 \\
\hline Angiotensinogen & Pan troglodytes & 1 & Serpin & ANGT_PANTR & 452 \\
\hline
\end{tabular}

analysed peptides represent only a subset of the peptides generated from the human gut metaproteome but are produced by meaningful members of the gut microbiota that, for instance, are decreased in the framework of inflammatory disorders. The potential effect of these peptides over the different nervous cells that are located in the gut is worthy of further deeper exploration and may lead to the interesting and timely relevant conclusion that the human gut microbiota, and most notably psychobiotics, might influence the host through the gutbrain axis by shedding neuroactive peptides.

\section{Conflicts of interest}

Borja Sánchez is on the scientific board and is co-founder of Microviable Therapeutics SL. The other authors do not have competing interests.

\section{Acknowledgment}

This work was supported by the Spanish "Programa Estatal de Investigación, Desarrollo e Inovación Orientada a los Retos de la Sociedad" (Grant AGL2016-78311-R); the Asociación Española Contra el Cancer ("Obtención de péptidos bioactivos contra el Cáncer ColoRectal a partir de secuencias genéticas de microbiomas intestinales”, grant PS-2016). This study was also supported by the Portuguese Foundation for Science and Technology (FCT) under the scope of the strategic funding of UID/BIO/04469/2013 unit and COMPETE 2020 (POCI-01-0145-FEDER006684). SING group thanks CITI (Centro de Investigación, Transferencia e Innovación) from University of Vigo for hosting its IT infrastructure. This work was partially supported by the Asturias Regional Plan I+D+i for research groups (FYCYT-IDI/2018/
000236) and by the Consellería de Educación, Universidades e Formación Profesional (Xunta de Galicia) under the scope of the strategic funding of ED431C2018/55-GRC Competitive Reference Group.

\section{Appendix A. Supplementary data}

Supplementary data to this article can be found online at https:// doi.org/10.1016/j.foodres.2019.01.069.

\section{References}

Achari, A. E., \& Jain, S. K. (2017). Adiponectin, a therapeutic target for obesity, diabetes, and endothelial dysfunction. International Journal of Molecular Sciences.. https://doi. org/10.3390/ijms18061321.

Atarashi, K., Tanoue, T., Oshima, K., Suda, W., Nagano, Y., Nishikawa, H., ... Honda, K. (2013). Treg induction by a rationally selected mixture of Clostridia strains from the human microbiota. Nature, 500, 232-236. https://doi.org/10.1038/nature12331.

Bailey, T. L., Boden, M., Buske, F. A., Frith, M., Grant, C. E., Clementi, L., ... Noble, W. S. (2009). MEME SUITE: Tools for motif discovery and searching. Nucleic Acids Research, 37(Web Server), W202-W208. https://doi.org/10.1093/nar/gkp335.

Bedoui, S., von Hörsten, S., \& Gebhardt, T. (2007). A role for neuropeptide Y (NPY) in phagocytosis: Implications for innate and adaptive immunity. Peptides, 28(2), 373-376. https://doi.org/10.1016/j.peptides.2006.07.029.

Blanco-Míguez, A., Gutiérrez-Jácome, A., Fdez-Riverola, F., Lourenço, A., \& Sánchez, B. (2017). MAHMI database: A comprehensive MetaHitbased resource for the study of the mechanism of action of the human microbiota. Database, 2017(1), https://doi. org /10.1093/database/baw157.

Blander, J. M., Longman, R. S., Iliev, I. D., Sonnenberg, G. F., \& Artis, D. (2017). Regulation of inflammation by microbiota interactions with the host. Nature Immunology. https://doi.org/10.1038/ni.3780.

Burbach, J. P. H. (2010). Neuropeptides from concept to online database www.neuropeptides.nl. European Journal of Pharmacology, 626(1), 27-48. https://doi.org/10. 1016/j.ejphar.2009.10.015.

Camacho, C., Coulouris, G., Avagyan, V., Ma, N., Papadopoulos, J., Bealer, K., \& Madden, T. L. (2009). BLAST + : Architecture and applications. BMC Bioinformatics, 10(1), 421. https://doi.org/10.1186/1471-2105-10-421. 
Chang, M. M., Leeman, S. E., \& Niall, H. D. (1971). Amino-acid sequence of substance P. Nature New Biology, 232(29), 86-87. https://doi.org/10.1038/newbio232086a0.

Cryan, J. F., \& Dinan, T. G. (2012). Mind-altering microorganisms: The impact of the gut microbiota on brain and behaviour. Nature Reviews Neuroscience, 13(10), 701-712. https://doi.org/10.1038/nrn3346.

Dimitrijević, M., \& Stanojević, S. (2013). The intriguing mission of neuropeptide Y in the immune system. Amino Acids, 45(1), 41-53. https://doi.org/10.1007/s00726-0111185-7.

Dinan, T. G., Stanton, C., \& Cryan, J. F. (2013). Psychobiotics: A novel class of psychotropic. Biological Psychiatry. https://doi.org/10.1016/j.biopsych.2013.05.001.

V Euler, U. S., \& Gaddum, J. H. (1931). An unidentified depressor substance in certain tissue extracts. The Journal of Physiology, 72(1), 74-87. Retrieved from http://www. ncbi.nlm.nih.gov/pubmed/16994201

Fantuzzi, G. (2005). Adipose tissue, adipokines, and inflammation. Journal of Allergy and Clinical Immunology.. https://doi.org/10.1016/j.jaci.2005.02.023.

Fonseca, V. (2003). Effect of thiazolidinediones on body weight in patients with diabetes mellitus. American Journal of Medicine. https://doi.org/10.1016/j.amjmed.2003.09. 005.

Fung, T. C., Olson, C. A., \& Hsiao, E. Y. (2017). Interactions between the microbiota, immune and nervous systems in health and disease. Nature Neuroscience, 20(2), 145-155. https://doi.org/10.1038/nn.4476.

Furusawa, Y., Obata, Y., Fukuda, S., Endo, T.a., Nakato, G., Takahashi, D., ... Ohno, H. (2013). Commensal microbe-derived butyrate induces the differentiation of colonic regulatory T cells. Nature, 504(7480), 446-450. https://doi.org/10.1038/ nature12721.

Guo, C.-J., Chang, F.-Y., Wyche, T. P., Backus, K. M., Acker, T. M., Funabashi, M., ... Fischbach, M. A. (2017). Discovery of Reactive Microbiota-Derived Metabolites that Inhibit Host Proteases. Cell, 168(3), 517-526.e18. https://doi.org/10.1016/j.cell. 2016.12.021.

Herbert, K. R., Williams, G. M., Cooper, G. J. S., \& Brimble, M. A. (2012). Synthesis of glycosylated 5-hydroxylysine, an important amino acid present in collagen-like proteins such as adiponectin. Organic \& Biomolecular Chemistry, 10(6), 1137. https:// doi.org/10.1039/c1ob06394d.

Hidalgo-Cantabrana, C., Moro-García, M. A., Blanco-Míguez, A., Fdez-Riverola, F. Lourenço, A., Alonso-Arias, R., \& Sánchez, B. (2017). In silico screening of the human gut metaproteome identifies Th17-promoting peptides encrypted in proteins of commensal bacteria. Frontiers in Microbiology, 8(SEP), https://doi.org/10.3389/ fmicb.2017.01726.

Holzer, P. (2016). Neuropeptides, microbiota, and behavior. 67-89. https://doi.org/10. 1016/bs.irn.2016.08.005.

Holzer, P., \& Farzi, A. (2014). Neuropeptides and the microbiota-gut-brain axis. 195-219. https://doi.org/10.1007/978-1-4939-0897-4_9.

Holzer, P., Reichmann, F., \& Farzi, A. (2012). Neuropeptide Y, peptide YY and pancreatic polypeptide in the gut-brain axis. Neuropeptides, 46(6), 261-274. https://doi.org/10. 1016/j.npep.2012.08.005

Kastin, A. J. (2013). In A. J. Kastin (Ed.). Handbook of biologically active peptides(2nd Edition). San Diego: Academic Press.

Kim, Y., Bark, S., Hook, V., \& Bandeira, N. (2011). NeuroPedia: neuropeptide database and spectral library. Bioinformatics, 27(19), 2772-2773. https://doi.org/10.1093/ bioinformatics/btr445.

Langille, M. G. I., Zaneveld, J., Caporaso, J. G., McDonald, D., Knights, D., Reyes, J. A., ... Huttenhower, C. (2013). Predictive functional profiling of microbial communities using 16S rRNA marker gene sequences. Nature Biotechnology, 31(9), 814-821. https://doi.org/10.1038/nbt.2676.

Lathrop, S. K., Bloom, S. M., Rao, S. M., Nutsch, K., Lio, C.-W., Santacruz, N., ... Hsieh, C.S. (2011). Peripheral education of the immune system by colonic commensal microbiota. Nature, 478(7368), 250-254. https://doi.org/10.1038/nature10434.

Lightfoot, Y. L., Selle, K., Yang, T., Goh, Y. J., Sahay, B., Zadeh, M., ... Mohamadzadeh, M. (2015). SIGNR 3 -dependent immune regulation by Lactobacillus acidophilus surface layer protein A in colitis. EMBO Journal, 34(November), 1-16. https://doi.org/10. 15252/embj.201490296.

Machiels, K., Joossens, M., Sabino, J., De Preter, V., Arijs, I., Eeckhaut, V., ... Vermeire, S. (2013). A decrease of the butyrate-producing species Roseburia hominis and
Faecalibacterium prausnitzii defines dysbiosis in patients with ulcerative colitis. Gut, 1-9. https://doi.org/10.1136/gutjnl-2013-304833.

Madden, T. (2013). The BLAST sequence analysis tool. Retrieved April 18, 2016, from http://www.ncbi.nlm.nih.gov/books/NBK153387/.

Matsuzawa, Y. (2010). Adiponectin: A key player in obesity related disorders. Current Pharmaceutical Design, 16(17), 1896-1901. Retrieved from http://www.ncbi.nlm.nih. gov/pubmed/20370675.

Mazmanian, S. K., Round, J. L., \& Kasper, D. L. (2008). A microbial symbiosis factor prevents intestinal inflammatory disease. Nature, 453(7195), 620-625. https://doi. org/10.1038/nature07008.

McDonald, D., Price, M. N., Goodrich, J., Nawrocki, E. P., DeSantis, T. Z., Probst, A., ... Hugenholtz, P. (2012). An improved Greengenes taxonomy with explicit ranks for ecological and evolutionary analyses of bacteria and archaea. The ISME Journal, 6(3), 610-618. https://doi.org/10.1038/ismej.2011.139.

Nicholson, J. K., Holmes, E., Kinross, J., Burcelin, R., Gibson, G., Jia, W., \& Pettersson, S. (2012). Host-Gut Microbiota Metabolic Interactions. Science, 336(6086), 1262-1267. https://doi.org/10.1126/science.1223813.

O'Reilly, M. S., Boehm, T., Shing, Y., Fukai, N., Vasios, G., Lane, W. S., ... Folkman, J. (1997). Endostatin: An endogenous inhibitor of angiogenesis and tumor growth. Cell, 88(2), 277-285 (9008168).

Pruitt, K. D., Tatusova, T., Brown, G. R., \& Maglott, D. R. (2012). NCBI Reference Sequences (RefSeq): Current status, new features and genome annotation policy. Nucleic Acids Research, 40. https://doi.org/10.1093/nar/gkr1079 (Database issue), D130-5.

Rao, M., \& Gershon, M. D. (2016). The bowel and beyond: The enteric nervous system in neurological disorders. Nature Reviews Gastroenterology and Hepatology.. https://doi. org/10.1038/nrgastro.2016.107.

Rooks, M. G., \& Garrett, W. S. (2016). Gut microbiota, metabolites and host immunity. Nature Reviews Immunology. https://doi.org/10.1038/nri.2016.42.

Sathyabama, S., Khan, N., \& Agrewala, J. N. (2014). Friendly pathogens: Prevent or provoke autoimmunity. Critical Reviews in Microbiology, 40(3), 273-280. https://doi org/10.3109/1040841X.2013.787043.

Shetty, S., Kusminski, C. M., \& Scherer, P. E. (2009). Adiponectin in health and disease: Evaluation of adiponectin-targeted drug development strategies. Trends in Pharmacological Sciences, 30(5), 234-239. https://doi.org/10.1016/j.tips.2009.02. 004.

Sokol, H., Pigneur, B., Watterlot, L., Lakhdari, O., Bermúdez-Humarán, L. G., Gratadoux, J.-J., ... Langella, P. (2008). Faecalibacterium prausnitzii is an anti-inflammatory commensal bacterium identified by gut microbiota analysis of Crohn disease patients. Proceedings of the National Academy of Sciences of the United States of America, 105(43), 16731-16736. https://doi.org/10.1073/pnas.0804812105.

Strand, F. L. (1999). Neuropeptides. Regulators of physiological processes. Cambridge: MIT Press.

Trujillo, M. E., \& Scherer, P. E. (2005). Adiponectin - Journey from an adipocyte secretory protein to biomarker of the metabolic syndrome. Journal of Internal Medicine, 257(2), 167-175. https://doi.org/10.1111/j.1365-2796.2004.01426.x.

UniProt Consortium, T. (2018). UniProt: The universal protein knowledgebase. Nucleic Acids Research, 46(5), 2699. https://doi.org/10.1093/nar/gky092.

Wang, Y., Lam, K. S. L., Xu, J. Y., Lu, G., Xu, L. Y., Cooper, G. J. S., \& Xu, A. (2005). Adiponectin inhibits cell proliferation by interacting with several growth factors in an oligomerization-dependent manner. Journal of Biological Chemistry, 280(18), 18341-18347. https://doi.org/10.1074/jbc.M501149200.

Wang, Y., Wang, M., Yin, S., Jang, R., Wang, J., Xue, Z., \& Xu, T. (2015). NeuroPep: A comprehensive resource of neuropeptides. Database, 2015https://doi.org/10.1093/ database/bav038 bav038-bav038.

Wang, Y., Xu, A., Knight, C., Xu, L. Y., \& Cooper, G. J. S. (2002). Hydroxylation and glycosylation of the four conserved lysine residues in the collagenous domain of adiponectin. Journal of Biological Chemistry, 277(22), 19521-19529. https://doi.org/ 10.1074/jbc.M200601200.

Wheway, J., Herzog, H., \& Mackay, F. (2007). NPY and receptors in immune and inflammatory diseases. Current Topics in Medicinal Chemistry, 7(17), 1743-1752. Retrieved from http://www.ncbi.nlm.nih.gov/pubmed/17979783. 